\title{
Thermal Radiation Equilibrium: (Nonrelativistic) Classical Mechanics versus (Relativistic) Classical Electrodynamics
}

\author{
Timothy H. Boyer \\ Department of Physics, City College of the City \\ University of New York, New York, New York 10031
}

\begin{abstract}
Physics students continue to be taught the erroneous idea that classical physics leads inevitably to energy equipartition, and hence to the Rayleigh-Jeans law for thermal radiation equilibrium. Actually, energy equipartition is appropriate only for nonrelativistic classical mechanics, but has only limited relevance for a relativistic theory such as classical electrodynamics. In this article, we discuss harmonic-oscillator thermal equilibrium from three different perspectives. First, we contrast the thermal equilibrium of nonrelativistic mechanical oscillators (where point collisions are allowed and frequency is irrelevant) with the equilibrium of relativistic radiation modes (where frequency is crucial). The Rayleigh-Jeans law appears from applying a dipole-radiation approximation to impose the nonrelativistic mechanical equilibrium on the radiation spectrum. In this discussion, we note the possibility of zero-point energy for relativistic radiation, which possibility does not arise for nonrelativistic classical-mechanical systems. Second, we turn to a simple electromagnetic model of a harmonic oscillator and show that the oscillator is fully in radiation equilibrium (which involves all radiation multipoles, dipole, quadrupole, etc.) with classical electromagnetic zero-point radiation, but is not in equilibrium with the Rayleigh-Jeans spectrum. Finally, we discuss the contrast between the flexibility of nonrelativistic mechanics with its arbitrary potential functions allowing separate scalings for length, time, and energy, with the sharply-controlled behavior of relativistic classical electrodynamics with its single scaling connecting together the scales for length, time, and energy. It is emphasized that within classical physics, energy-sharing, velocity-dependent damping is associated with the low-frequency, nonrelativistic part of the Planck thermal radiation spectrum, whereas acceleration-dependent radiation damping is associated with the high-frequency adiabatically-invariant and Lorentz-invariant part of the spectrum corresponding to zero-point radiation.
\end{abstract}




\section{INTRODUCTION}

\section{A. Erroneous Textbook Claim}

The current textbooks of modern physics still make the erroneous claim that classical physics leads inevitably to the Rayleigh-Jeans spectrum for thermal radiation equilibrium. [1] Indeed the treatment of classical physics in these texts seems to have progressed only so far as James Jeans' Report on Radiation and the Quantum-Theory in 1914, but no further.[2] In this article, we make another[3] attempt to counteract the erroneous textbook claim. Specifically, we point out that a simple electromagnetic-model harmonic oscillator is not in equilibrium with the Rayleigh-Jeans spectrum. In the discussion, we emphasize the contrasting points of view between nonrelativistic classical mechanics and relativistic classical electrodynamics in connection with equilibrium for harmonic oscillators.

Our earlier attempt[3] to correct the misinformation regarding thermal radiation equilibrium in classical physics involved an extended historical survey. It was pointed out that the introduction of classical electromagnetic zero-point radiation led to modifications of the historical arguments. The modified arguments provide natural classical explanations for the Planck spectrum within relativistic classical physics. The Planck spectrum with zero-point radiation corresponds to a radiation energy per normal mode

$$
U_{\text {rad }}(\omega, T)=\frac{1}{2} \hbar \omega \operatorname{coth}\left(\frac{\hbar \omega}{2 k_{B} T}\right)=\frac{\hbar \omega}{\exp \left[\hbar \omega /\left(k_{B} T\right)\right]-1}+\frac{1}{2} \hbar \omega .
$$

The spectrum involves a transition from the relativistic high-frequency region, $\hbar \omega /\left(k_{B} T\right)>>1$, associated with adiabatically-invariant and Lorentz-invariant zero-point radiation where $U_{\text {rad }}(\omega, T) \rightarrow(1 / 2) \hbar \omega$, over to the low-frequency Rayleigh-Jeans region, $\hbar \omega /\left(k_{B} T\right)<<1$, associated with nonrelativistic energy-sharing behavior where $U_{\text {rad }}(\omega, T) \rightarrow k_{B} T$.

\section{B. Three Analyses for Radiation Equilibrium}

The present discussion is quite different from the previous historical survey. Here we present three different analyses of radiation equilibrium connected to harmonic oscillator systems; these analyses include aspects of point-particle collisions, adiabatic invariance, scattering, and scaling which do not appear in earlier work. 


\section{Point-Particle Collisions and Adiabatic Invariance}

After some basic preliminaries, we start our analysis by contrasting the equilibrium for two different situations; one is a box containing a finite number of mechanical oscillators connected through point collisions with a free particle, and the other involves a charged mechanical oscillator coupled to a divergent number of electromagnetic radiation modes. When the equipartition ideas of nonrelativistic mechanics are imposed upon radiation treated

in the dipole approximation, the Rayleigh-Jeans spectrum appears. Our analysis also suggests the possibility of classical zero-point energy for radiation associated with oscillator adiabatic invariance, something which is not possible for classical mechanical systems which allow point-particle collisions.

\section{Equilibrium Under Scattering}

In the second equilibrium analysis, we emphasize that a charged harmonic oscillator connected to radiation through the dipole approximation can come to equilibrium with any radiation spectrum. In order to obtain a specific radiation spectrum, some additional assumptions are needed. The Rayleigh-Jeans spectrum and zero-point spectrum correspond to the extremes allowed by Wien's displacement theorem. If we turn to invariance under scattering as our criterion for a preferred spectrum, then again there are two natural possibilities. In one possibility, the mechanical behavior is modified to an anharmonic nonrelativistic oscillator while maintaining the dipole approximation to radiation. The other possibility involves maintaining the harmonic mechanical behavior but going to electromagnetic interactions beyond the dipole approximation, giving an approximately relativistic oscillator system. We point out that the first nonrelativistic procedure leads to the Rayleigh-Jeans spectrum, while the second relativistic analysis leads to the classical zero-point radiation spectrum. For a harmonic oscillator, the Rayleigh-Jeans spectrum arising from the energy equipartition ideas of nonrelativistic classical mechanics is not tolerated beyond the dipole coupling to radiation. 


\section{Scaling in Nonrelativistic Mechanics and in Relativistic Electrodynamics}

Finally, in the third equilibrium analysis, we point out the sharp differences in scaling behavior between nonrelativistic mechanics and relativistic electrodynamics, and connect these differences to the requirements for thermal equilibrium. The enormous flexibility of nonrelativistic classical mechanics contrasts with the strictly-controlled radiation connections of relativistic classical electrodynamics. And this contrast is crucial in questions of thermal radiation equilibrium. We emphasize that, within classical physics, the full Planck spectrum requires both velocity-dependent damping and acceleration-dependent radiation damping.

\section{Missing Aspects in the Physics of 1900: Zero-Point Radiation and Relativity}

The foundation for the erroneous claims regarding classical thermal radiation which pervade the textbooks and the internet lies in the fact that the physicists of the early 20th century were (just as the physicists of today are) unaware of two crucial aspects of classical physics existing within classical electrodynamics: 1)the existence of classical electromagnetic zero point radiation, and 2)the importance of special relativity. In this article, we illustrate the importance of these two aspects of classical physics by comparing the ideas of thermal equilibrium which arise in classical mechanics and in classical electrodynamics. The models for our discussion are harmonic oscillators.

\section{BASIC PRELIMINARIES}

\section{A. Some Contrasts Between (Nonrelativistic) Classical Mechanics and ( Relativis-} tic) Classical Electrodynamics.

In order to prepare the reader for our analysis, we first mention some of the important contrasts between classical mechanics and classical electrodynamics. Classical mechanics allows point collisions between massive particles which provide velocity-dependent damping, whereas classical electrodynamics involves only long-range forces associated with electromagnetic fields. Classical mechanics allows particles to exist without any connection to a spacetime-dependent field, whereas in classical electrodynamics, every charged particle is 
connected to electromagnetic fields which introduce acceleration-dependent radiation forces. There is no special role for oscillation frequency when considering thermal equilibrium within nonrelativistic classical mechanics, whereas classical electrodynamics determines thermal equilibrium through the frequency-dependent connection of charges to radiation fields. Finally, the relativistic theory of classical mechanical particles is an insignificant part of mechanics; the relativistic theory is restricted to point collisions between particles and allows no potential functions. [4] Classical mechanics which allows the interaction of particles beyond point collisions satisfies Galilean symmetry, whereas classical electrodynamics satisfies Lorentz symmetry.

\section{B. Harmonic Oscillator in Action-Angle Variables}

A one-dimensional harmonic oscillator of natural (angular) frequency $\omega_{0}$ and mass $m$ can be described by the Hamiltonian

$$
H(x, p)=p^{2} /(2 m)+(1 / 2) m \omega_{0}^{2} x^{2}=U_{o s c} .
$$

Within nonrelativistic mechanics, this Hamiltonian will give the equations of motion for the position $x$ and momentum $p$ while the initial conditions will determine the actual motion, including the energy $U_{\text {osc }}$ and phase of the oscillation. When discussing adiabatic changes in the oscillator's natural frequency $\omega_{0}$, it is convenient to reexpress the Hamiltonian in terms of action-angle variables $w$ and $J$ using the canonical transformation with the connection

$$
x=\sqrt{\frac{2 J}{m \omega_{0}}} \sin w \text { and } p=\sqrt{2 m \omega_{0} J} \cos w .
$$

In terms of the new variables $w$ and $J$, the Hamiltonian takes the form

$$
H=\frac{p^{2}}{2 m}+\frac{1}{2} m \omega_{0}^{2} x^{2}=J \omega_{0}=U_{o s c}
$$

In terms of action-angle variables, the oscillator Hamiltonian no longer refers to the particle mass $m$ nor the amplitude of oscillation $x$. The initial conditions again give the oscillator energy $U_{o s c}=J \omega_{0}$ and the initial phase $\phi$ in $w=\omega_{0} t+\phi$. 


\section{Thermodynamics of the Harmonic Oscillator and Wien's Theorem}

A harmonic oscillator (whether a mechanical oscillator or a radiation mode) is such a simple system that its thermodynamic behavior depends upon only two variables, its natural frequency $\omega_{0}$ and its temperature $T$. By considering the adiabatic change of the oscillator frequency $\omega_{0}$, it is easy to derive Wien's displacement theorem indicating that in thermal equilibrium, the energy of the oscillator must take the form [5]

$$
U_{o s c}\left(\omega_{0}, T\right)=\omega_{0} f\left(\omega_{0} / T\right)
$$

where $f\left(\omega_{0} / T\right)$ is an unknown function of the ratio $\omega_{0} / T$. The extremes of energy equipartition and zero-point energy correspond to the limiting situations where the energy $U_{\text {osc }}\left(\omega_{0}, T\right)$ becomes independent of one of the two variables. Thus if $f\left(\omega_{0} / T\right) \rightarrow$ const $\times\left(T / \omega_{0}\right)$, then we have energy equipartition $U_{\text {osc }}\left(\omega_{0}, T\right) \rightarrow$ const $\times T$; on the other hand, if $f\left(\omega_{0} / T\right) \rightarrow$ const ${ }^{\prime}$, then one finds temperature-independent zero-point energy $U_{\text {osci }}\left(\omega_{0}, T\right) \rightarrow$ const $^{\prime} \times \omega_{0}$. If we consider only the first two laws of thermodynamics, then the function $f\left(\omega_{0} / T\right)$ giving the transition between these extremes is unknown. Only if we add further criteria such as "smoothness" [5] or inclusion of the third law, [6] do we obtain the Planck spectrum with zero-point energy given in Eq. (11). However, both these additional criteria are not currently in fashion in physics, and below we will consider other possibilities.

\section{Thermal Radiation Modes}

The solutions of Maxwell's equations involve electromagnetic fields arising both from charge and current sources, and also from boundary conditions. The boundary-condition electromagnetic fields satisfy the source-free Maxwell equations. Thermal radiation can be treated as boundary-condition radiation, and can be expanded in terms of plane waves with

random phases satisfying periodic boundary conditions in a large cubic box with sides of length $a$,

$$
\begin{gathered}
\mathbf{E}(\mathbf{r}, t)=\sum_{\mathbf{k}, \lambda} \widehat{\epsilon}(\mathbf{k}, \lambda)\left(\frac{8 \pi U_{\operatorname{rad}}(\omega)}{a^{3}}\right)^{1 / 2} \frac{1}{2}\{\exp [i \mathbf{k} \cdot \mathbf{r}-i \omega t+i \theta(\mathbf{k}, \lambda)]+c c\}, \\
\mathbf{B}(\mathbf{r}, t)=\sum_{\mathbf{k}, \lambda} \widehat{\mathbf{k}} \times \widehat{\epsilon}(\mathbf{k}, \lambda)\left(\frac{8 \pi U_{r a d}(\omega)}{a^{3}}\right)^{1 / 2} \frac{1}{2}\{\exp [i \mathbf{k} \cdot \mathbf{r}-i \omega t+i \theta(\mathbf{k}, \lambda)]+c c\},
\end{gathered}
$$


where the sum over the wave vectors $\mathbf{k}=\widehat{x} 2 \pi l / a+\widehat{y} 2 \pi m / a+\widehat{z} 2 \pi n / a$ involves integers $l, m, n=0, \pm 1, \pm 2, \ldots$ running over all positive and negative values, there are two polarizations $\widehat{\epsilon}(\mathbf{k}, \lambda), \lambda=1,2$, and the random phases $\theta(\mathbf{k}, \lambda)$ are distributed uniformly over the interval $(0,2 \pi]$, independently for each wave vector $\mathbf{k}$ and polarization $\lambda$. The notation " $c c$ " refers to the complex conjugate. We have assumed that the radiation spectrum is isotropic, and that the energy per normal mode at radiation frequency $\omega=c k$ is given by $U_{\text {rad }}(\omega)$. There are a divergent number of radiation normal modes with no limit on the frequency $\omega$.

\section{E. Equilibrium Between a Charged Oscillator and Random Radiation in the}

\section{Dipole Approximation}

When treated in the dipole approximation, the interaction of a charged harmonic oscillator with the random radiation in Eqs. (6) and (77) can be written as Newton's second law

$$
m \ddot{x}=-m \omega_{0}^{2} x+m \tau \dddot{x}+e E_{x}(0, t),
$$

involving the harmonic restoring force $-m \omega_{0}^{2} x$, the dipole radiation damping force $m \tau \dddot{x}$ with $\tau=2 e^{2} /\left(3 m c^{3}\right)$, and the radiation driving force treated in dipole approximation $e E_{x}(0, t)$. This equation of motion has been solved many times, beginning with Planck's work at the end of the 19th century. [7] [8] [9] [10] It is found that in this dipole approximation, the oscillator acts essentially like a radiation mode at the oscillation frequency $\omega_{0}$ : the average energy of the oscillator matches the average energy of the radiation modes at frequency $\omega_{0}$, $U_{\text {osc }}\left(\omega_{0}\right)=U_{\text {rad }}\left(\omega_{0}\right)$, and the phase space distribution $P_{\text {osc }}(x, p)$ of the oscillator takes the form

$$
P_{\text {osc }}(x, p)=\text { const } \times \exp \left[-H(x, p) / U_{\text {rad }}\left(\omega_{0}\right)\right]
$$

where $H(x, p)$ is the Hamiltonian of the oscillator given in Eqs. (2) or (4).

In the dipole approximation, the harmonic oscillator is in equilibrium with any spectrum $U_{\text {rad }}(\omega)$ of isotropic random radiation. Thus, other than the requirement of isotropy in direction, the spectrum $U_{\text {rad }}(\omega)$ is arbitrary. The oscillator will enforce isotropic behavior for the radiation spectrum, but the harmonic oscillator will not change the frequency

distribution of radiation among the various frequencies. [9] 


\section{EXPLORING EQUILIBRIUM FOR OSCILLATORS IN MECHANICS AND IN ELECTROMAGNETIC RADIATION}

We now turn from the preliminary aspects of our analysis over to specific treatments of radiation equilibrium for harmonic oscillators.

\section{A. Equilibrium for Systems of Oscillators}

Suppose that we have an uncharged free particle of mass $M$ and a special harmonic oscillator of (angular) frequency $\omega_{0}$ at our disposal. We wish to contrast the equilibrium behavior for two different systems. The first system involves a collection of $N$ non-interacting, classical-mechanical, one-dimensional oscillators of various frequencies $\omega$ in an elastic-walled box; the second involves the electromagnetic radiation in a reflecting-walled box. Both boxes can be described as containing harmonic oscillators inside, the one in terms of mechanical oscillators and the other in terms of electromagnetic radiation modes. We assume that the oscillators in both boxes are in "thermal equilibrium," and we wish to explore this thermal equilibrium.

\section{B. Equilibrium for Mechanical Oscillators}

\section{Equilibrium Through Point-Particle Collisions}

If we introduce our special oscillator into the box of $N$ mechanical oscillators, the oscillator will not come to equilibrium in the box unless there is some interaction between this oscillator and the original oscillators in the box. However, if we now introduce our free particle $M$ into the box of mechanical oscillators, then the particle will collide with all the masses of the mechanical oscillators and will bring to equilibrium the entire collection (including both our special oscillator, the $N$ oscillators in the box, and also the mass $M$ ) by sharing the total system energy $U_{\text {total }}$ among the finite number $N+1$ of mechanical oscillators and the mass M. Each oscillator of mechanical frequency $\omega$ will come to equilibrium at the Boltzmann distribution $P_{\text {osc }}(J, \omega)=$ const $\times \exp \left[-J \omega / U_{a v}\right]$ where here $J$ is the action variable of the oscillator with Hamiltonian $H=J \omega$, and $U_{a v}=U_{\text {total }} /(N+1+3 / 2)$ is the average energy, (from energy equipartition) with the average energy of each one-dimensional oscillator $2 / 3$ 
that of the mass $M$ which has three-dimensional motion. We notice that the natural frequency $\omega$ of an oscillator is irrelevant for the average oscillator energy at equilibrium; our special oscillator at frequency $\omega_{0}$ has the same average energy as any oscillator at any frequency $\omega$. However, we notice that the phase space distribution $P_{\text {osc }}(J, \omega)$ for an oscillator does depend upon its frequency $\omega$ and also upon the average energy $U_{a v}$ for the entire system.

\section{Change of Equilibrium Due to Adiabatic Change of Oscillator Frequency}

If we now isolate our special oscillator and carry out an adiabatic change in the frequency, the quantity of energy divided by frequency, $U_{a v} / \omega_{0}=J$, is an adiabatic invariant and does not change. When the frequency is increased from $\omega_{0}$ to $\omega_{0}^{\prime}$, the energy of our special oscillator is increased from $U_{a v}$ over to $U^{\prime}=\left(\omega_{0}^{\prime} / \omega_{0}\right) U_{a v}$. This energy $U^{\prime}$ is now above the average energy $U_{a v}$ of the other oscillators in the box. On reconnecting our special oscillator to the collection of the other mechanical oscillators and the mass $M$, the average energy of each oscillator will increase, and the phase space distribution of each oscillator will change to give a larger average value of its action variable $J$. Thus an adiabatic change in the frequency of our special oscillator will indeed disturb the equilibrium of the entire system.

\section{Equilibrium for Random Radiation}

\section{Charged-Oscillator Equilibrium Depends on Oscillator Frequency}

This thermal-equilibrium situation for nonrelativistic mechanical oscillators (where pointparticle collisions are allowed, and there is no particular role regarding energy equilibrium for the oscillator frequency) is to be contrasted with the electromagnetic situation which involves a divergent number of massless time-harmonic radiation modes characterized by frequency. In analogy with the mechanical case, the introduction of a special harmonic oscillator of frequency $\omega_{0}$ into a conducting-walled enclosure gives us no information unless we specify the

interaction of the oscillator with the radiation modes of the enclosure. A free but uncharged particle $M$ has no interaction with the electromagnetic oscillators. However, we can connect our special mechanical oscillator to radiation by taking the oscillating particle as charged. As pointed out by Planck at the end of the 19th century and noted above in our preliminaries, treated in the dipole approximation, this electromagnetic harmonic oscillator will interact 
with the radiation modes at the same frequency as the natural frequency of the oscillator and will come to equilibrium with these modes, but not with modes of differing frequency. Thus the frequency-dependent connection between an oscillator and electromagnetic radiation is a crucial aspect of electromagnetism.

\section{Possibility of Adiabatic Invariance in Zero-Point Radiation}

We now consider an adiabatic change in the frequency $\omega_{0}$ of our oscillator over to a new frequency $\omega_{0}^{\prime}$. During this adiabatic change, the ratio of energy to frequency, $U_{0} / \omega_{0}=J$, of our special oscillator is unchanged. We can now have our oscillator (treated in the dipole approximation) interact with radiation at the new frequency $\omega_{0}^{\prime}$. If the radiation modes at $\omega_{0}^{\prime}$ have greater energy than our oscillator, then, on interaction, there will be energy transferred from the radiation modes at $\omega_{0}^{\prime}$ over to our special oscillator. On the other hand, if the radiation modes at $\omega_{0}^{\prime}$ have less energy, then the transfer is reversed. However, we also have the possibility that our special oscillator has the same average energy as the radiation modes at $\omega_{0}^{\prime}$ and so is already in equilibrium with these modes. This situation of undisturbed equilibrium despite an adiabatic change of an oscillator's frequency and energy is an entirely new possibility which does not arise for mechanical oscillators where energy equipartition is involved. Here in the electromagnetic situation, we have the possibility of making adiabatic changes in the frequency and energy of our oscillator and yet not transferring any average energy between the electromagnetic radiation modes. If this situation of thermal equilibrium holds for all radiation modes, then the classical electromagnetic spectrum is Lorentz invariant and is termed "classical electromagnetic zeropoint radiation." [1]

\section{Introduction of Planck's Constant $\hbar$}

The scale of this zero-point radiation involves the ratio $U / \omega$ which is the same for all radiation frequencies. The scale which fits with nature corresponds to the introduction of Planck's constant $\hbar$ as the scale invariant so that the average energy $U_{r a d}(\omega)$ of a radiation mode of frequency $\omega$ satisfies $U_{\text {rad }}(\omega)=(1 / 2) \hbar \omega$. There is no question that, when interpreted in terms of classical physics, nature contains classical electromagnetic zero-point 
radiation. Thus the introduction of Planck's constant $\hbar$ as the scale of classical zero-point radiation gives fully classical explanations for Casimir forces, van der Waals forces, oscillator

specific heats, diamagnetism, and the Planck spectrum of thermal radiation.[12] Zero-point radiation involves the same phase space distribution for every radiation mode no matter what its frequency,

$$
P_{\text {rad zp }}(J)=\text { const } \times \exp \left[-\frac{J \omega}{(1 / 2) \hbar \omega}\right]=\text { const } \times \exp \left[-\frac{J}{\hbar / 2}\right] .
$$

\section{Planck's Constant $\hbar$ Can Appear in Classical or Quantum Theories}

The appearance of Planck's constant $\hbar$ within classical physics provokes the indignation of some physicists. Some uninformed physicists continue to insist that Planck's constant $\hbar$ is a "quantum constant," and that any theory in which $\hbar$ appears has a "quantum" element. That this claim is untrue has been pointed out in work published in the American Journal of Physics.[13] Actually, Planck's constant $\hbar$ is a physical constant, like Cavendish's constant $G$, and can appear in any theory which involves an appropriate scale, just as the constant $G$ can appear in both Newtonian gravity and in general relativity since $G$ sets the scale of the gravitational interaction. In modern theory, Planck's constant appears both as the scale of action in quantum theory and as the scale of classical zero-point radiation in classical electrodynamics.

Because Planck's constant $\hbar$ does not appear in Maxwell's equations but only in the source-free boundary condition[13] on the equations, there are two natural versions of classical electrodynamics, one including $\hbar$ and one not. The version which includes Planck's constant provides a natural classical explanation of the Planck spectrum[3] and of some other phenomena [12] with a scale set by $\hbar$. The version which appears in current textbooks of classical electromagnetism assumes that the source-free electromagnetic field vanishes and so offers no explanation of phenomena at the scale of $\hbar$.

It remains true that Planck's constant $\hbar$ has no place in classical mechanics because classical mechanics (with point-particle collisions which share all energy) allows no zeropoint energy. Of course, classical mechanics also does not allow massless waves which carry energy and momentum. However, classical electromagnetism is quite different from classical 
mechanics. Classical electromagnetism includes massless waves, and, indeed, has a natural place for Planck's constant as the scale of classical electromagnetic zero-point radiation.

\section{RADIATION EQUILIBRIUM FOR AN OSCILLATING CHARGED SYSTEM}

\section{A. A Point Harmonic Oscillator Does Not Determine Radiation Equilibrium}

In the discussion above, we have seen the reappearance of the same two oscillator energies (equipartition and zero-point energy) which appeared from the limits of Wien's law (55), arising from the thermodynamics of the harmonic oscillator. Again it must be emphasized that since a charged harmonic oscillator treated in the dipole approximation comes to equilibrium with any radiation spectrum, the determination of the actual spectrum of thermal radiation equilibrium must be based upon other factors. Here we list several criteria.

\section{B. Specific Criteria for Radiation Equilibrium}

\section{Adoption of the Nonrelativistic Oscillator Equilibrium}

In the early years of the 20th century, just as in the modern physics texts of today, it was assumed[1] [2] that nonrelativistic statistical mechanics correctly determines the phase space of the harmonic oscillator according to the Boltzmann distribution

$$
P_{\text {osc }}(x, p)=\text { const } \times \exp \left[-H(x, p) /\left(k_{B} T\right)\right]
$$

Then comparing equations (9) and (11), it was concluded that the spectrum of random radiation in equilibrium with the oscillator must be $U_{\text {rad }}(\omega)=k_{B} T$. In other words, nonrelativistic classical mechanics, which uses Boltzmann statistical mechanics, must lead to the Rayleigh-Jeans spectrum for relativistic classical radiation.

In 1910, Einstein and Hopf[14] [15] tried to avoid full Boltzmann statistical mechanics and to use instead only the well-established average thermal kinetic energy of a massive nonrelativistic particle in one dimension as $(1 / 2) M v^{2}=(1 / 2) k_{B} T$. However, they again arrived at the Rayleigh-Jeans spectrum for thermal radiation. 


\section{Adoption of the Spectrum Invariant Under Adiabatic Change}

An entirely different criterion involves adiabatic invariance. We saw above that, under an adiabatic change of frequency, a charged harmonic oscillator of frequency $\omega_{0}$ and energy $U\left(\omega_{0}\right)$ (when treated in the dipole approximation) will not transfer energy among radiation modes if the radiation spectrum is Lorentz invariant. Thus the assumption of adiabatic invariance for the oscillator picks out a special radiation spectrum, that of classical electromagnetic zero-point radiation. This is also the spectrum allowed by the limit of Wien's law (5) which makes the equilibrium spectrum independent of the temperature $T$.

\section{Adoption of the Spectrum Invariant Under Scattering}

A third criterion involves invariance under scattering. The charged harmonic oscillator treated in the dipole approximation scatters radiation so as to make the radiation pattern isotropic; however, the scattering by the oscillator does not change the frequency spectrum of the random radiation. In order to obtain a preferred spectrum based upon invariance under scattering, we must go beyond use of the harmonic oscillator treated in the dipole approximation.

a. Two Oscillator Extensions Leading to Equilibrium Radiation Spectra There are two natural extensions of the harmonic oscillator model. The first possible extension retains the electromagnetic aspect (continuing the use of the dipole approximation), but changes the mechanics (going beyond the harmonic oscillator over to an anharmonic oscillator). The second possible extension retains the mechanics (continuing the use of a purely harmonic mechanical oscillator), but changes the electromagnetic connection (going beyond the dipole radiation approximation over to full electromagnetic interaction at all harmonics). The two possible extensions give different preferred equilibrium spectra. The nonrelativistic mechanical extension while retaining the dipole radiation approximation gives the RayleighJeans spectrum. The relativistic electromagnetic extension while retaining the harmonic mechanical behavior gives classical zero-point radiation. Both extensions will be discussed below. 


\section{Radiation Scattering by an Anharmonic Nonrelativistic Mechanical System in} the Dipole Approximation

In order to avoid the use of the ideas of classical statistical mechanics, various physicists have considered the transition beyond harmonic oscillators to nonrelativistic anharmonic oscillators which will scatter radiation and change the spectrum of the random radiation. In 1924, Van Vleck[16] considered (and partially published) a general analysis of nonlinear nonrelativistic mechanical systems in scattering equilibrium where the radiation was treated in the dipole approximation. Work by other authors has considered scattering by a nonrelativistic nonlinear oscillator, [17] and scattering by nonrelativistic potentials where the charged particle momentum took relativistic form.[18] All of these classical scattering calculations arrived at the Rayleigh-Jeans spectrum. None of these calculations treats a relativistic scattering system.

A nonrelativistic anharmonic oscillator with a nonlinear term in energy $\alpha x^{3}$ and Hamiltonian

$$
H(x, p)=p^{2} /(2 m)+(1 / 2) m \omega_{0}^{2} x^{2}+\alpha x^{3}
$$

provides a simple example of a scattering system which will enforce the Rayleigh-Jeans spectrum for radiation equilibrium. The equation of motion takes the form

$$
m \ddot{x}=-m \omega_{0}^{2} x+3 \alpha x^{2}+m \tau \dddot{x}+e E_{x}(0, t),
$$

and continues the use of the dipole approximation in the radiation damping $m \tau \dddot{x}$ and in the electromagnetic force $e E_{x}(0, t)$. The mechanical motion of the oscillator now involves a mechanical oscillation at frequency $\omega_{1}$ which is different from $\omega_{0}$ and which depends upon the constant $\alpha$ giving the scale of the nonlinear term. [19] The mechanical motion now includes the harmonics [19] of the frequency $\omega_{1}$

$$
x(t)=D_{0}+D_{1} \cos \left[\omega_{1} t+\phi_{1}\right]+D_{2} \cos \left[2 \omega_{1}+\phi_{2}\right]+\ldots
$$

Since the mechanical motion has oscillations at all the harmonics $n \omega_{1}$ of the fundamental mechanical frequency $\omega_{1}$, the nonlinear oscillator will interact with radiation in the dipole approximation at all the harmonics of the oscillation frequency $\omega_{1}$. This interaction with radiation will transfer energy among the radiation modes, and so will lead to a unique equilibrium spectrum. We note that the radiation equilibrium is determined by the nonrelativistic mechanical system and not by any properties of the radiation with which the 
system has minimal (dipole) interaction. In all treatments with nonrelativistic nonlinear mechanical scatterers connected to radiation through the dipole approximation, one finds the Rayleigh-Jeans spectrum as the unique spectrum of radiation equilibrium. When van Vleck[16] partially published his analysis in 1924, the work was regarded as confirming that the Rayleigh-Jeans spectrum was the appropriate equilibrium radiation spectrum expected within classical physics.

\section{Radiation Scattering by a Charged Harmonic Oscillator with Full Electro- magnetic Interactions}

\section{One-Dimensional Electromagnetic Harmonic Oscillator}

In order to emphasize electromagnetic interactions in contrast to arbitrary mechanical potentials, we mention here the possibility of a one-dimensional oscillator where the oscillating particle of mass $m$ and charge $e$ moves under purely electromagnetic forces. We imagine a charge $e$ which is constrained to move along the straight line between two charges $q$ (of the same sign as $e$ ) separated by a distance $l$. The charge $e$ will then oscillate in one dimension along the line between the charges $q$, but is in unstable equilibrium against motion to the side if the constraint were removed. For small oscillations, the mechanical motion of $e$ is harmonic with (angular) frequency $\omega_{0}=\left[32 \mathrm{eq} /\left(\mathrm{ml}^{3}\right)\right]^{1 / 2}$. For any finite amplitude of oscillation, there will be non-harmonic contributions from the electromagnetic forces on $e$ due to the charges $q$. However, as the amplitude of oscillation becomes ever smaller, the oscillation becomes ever-more-nearly harmonic. Furthermore, on transformation to a new inertial frame $S^{\prime}$, the electromagnetic system would have relativistic behavior in the limit of zero-oscillation amplitude at a new Lorentz-transformed oscillation frequency $\omega_{0}^{\prime}$.

\section{Relativistic Harmonic Oscillator}

If we adopt this one-dimensional electromagnetic model for the oscillating charge $e$ where the restoring force arises from the electrostatic field in the rest frame of the charges $q$, then the relativistic equation for the constrained motion for the charge $e$ in electromagnetic fields

is $d \mathbf{p} / d t=e \mathbf{E}(\mathbf{r}, t)$. In the approximation that the velocity of the charge $e$ relative to the 
charges $q$ is small compared to $c$, one can approximate the relativistic change in momentum as mass times acceleration, accurate through first order in the ratio $v / c$. Thus we have

$$
\frac{d \mathbf{p}}{d t}=\frac{d}{d t}\left[\frac{m \mathbf{v}}{\left(1-v^{2} / c^{2}\right)^{1 / 2}}\right]=\frac{d}{d t}\left[m \mathbf{v}\left(1+\frac{v^{2}}{2 c^{2}}+\ldots\right)\right] \approx \frac{d(m \mathbf{v})}{d t}
$$

provided that we can neglect terms of order $(v / c)^{2}$. Our electromagnetic oscillator can be treated as a relativistic system provided that its oscillation velocity is small. The oscillator system is fully relativistic only in the limit $v \rightarrow 0$.

\section{Radiation Equilibrium Beyond the Dipole Approximation}

In his textbook of classical electrodynamics, Jackson points out that [20] "Appreciable radiation in multiples of the fundamental [oscillatory frequency] can occur because of relativistic effects ... even though the components of velocity are truly sinusoidal, or it can occur if the components of the velocity are not sinusoidal, even though periodic." All of the previous scattering calculations have involved mechanical motions which are "not sinusoidal, even though periodic," as in our Eq. (14). Thus the harmonics appear in the purely mechanical motion of the charged particle. However, recent work by Huang and Batelaan[21] regarding absorption of radiation at harmonics due to relativistic effects suggested the possibility of determining radiation equilibrium not from nonrelativistic mechanical motions but rather from purely relativistic effects in classical electromagnetism.

In problem 14 in Chapter 14, Jackson[20] asks a student to calculate the radiation emitted at the harmonics $n \omega_{0}$ due to a purely sinusoidal motion at $\omega_{0}$

$$
x=D \cos \left[\omega_{0} t+\phi\right] .
$$

Thus the treatment of the multipole moments of the oscillating charge can be extended beyond the dipole term to include the quadrupole moment of the harmonic oscillator which will emit radiation at frequency $2 \omega_{0}$, and indeed can be extended to all higher multipoles. However, if the harmonic oscillator can emit quadrupole radiation at the second harmonic $2 \omega_{0}$ of the oscillator's natural frequency $\omega_{0}$, then it can also absorb energy at the second harmonic. The force on the oscillator must be extended beyond the dipole interaction $e E_{x}(0, t)$ to include the next term in the expansion of the true force $e E_{x}(x, t)$ as

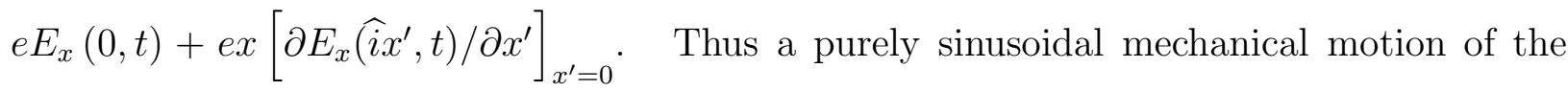


oscillator combined with the relativistic radiation analysis can lead to a specific equilibrium spectrum of random classical radiation. Here for the first time in the classical physics scattering literature, radiation equilibrium is determined not by nonrelativistic mechanical considerations but by relativistic electromagnetic aspects.

\section{Zero-Point Radiation as the Oscillator-Scattering Equilibrium Spectrum}

The calculation for the equilibrium radiation spectrum of a electromagnetic charged harmonic oscillator of small amplitude has been carried out.[22] The equilibrium spectrum is a Lorentz-invariant radiation spectrum. The Rayleigh-Jeans spectrum is not Lorentz invariant, and so is not the spectrum of radiation equilibrium for a charged classical harmonic oscillator when treated beyond the dipole approximation for the radiation interaction. Only Lorentz-invariant zero-point radiation will serve as an equilibrium spectrum.

\section{Use of Nonrelativistic versus Relativistic Theory}

A harmonic oscillator is like a clock with frequency $\omega_{0}$. The point harmonic oscillator allows only a dipole connection to radiation, and is in equilibrium with any spectrum of isotropic random radiation. In the dipole approximation, the radiation energy $U_{\text {rad }}\left(\omega_{0}\right)$ merely determines the one number giving the oscillator energy $U_{\text {osc }}\left(\omega_{0}\right)$. The point oscillator clock itself can assume either Galilean or Lorentz transformation properties when viewed in a new inertial frame. However, if the oscillator motion has finite amplitude, then the analysis of the oscillator must choose between the nonrelativistic and the relativistic transformations. If nonrelativistic mechanical motion beyond harmonic motion is involved, then there is enormous flexibility in the choice of the mechanical interaction potential $V(x)$; in equilibrium, the nonrelativistic nonlinear oscillator, with a dipole connection to radiation, enforces a frequency-independent constant energy on the radiation modes, corresponding to the frequency-independent energy $k_{B} T$ allowed by the Wien law (5). On the other hand, if relativistic electromagnetic interactions are assigned to the harmonic oscillator, then the extension from the dipole moment to the quadrupole moment and beyond allows no flexibility whatsoever; the harmonic oscillator motion is completely determined, and the equilibrium radiation spectrum assumes a Lorentz-invariant form, corresponding to the zero-point energy 
limit $(1 / 2) \hbar \omega_{0}$ allowed by the Wien law (5)

\section{The Relativistic Limit and Classical Zero-Point Radiation}

The electromagnetic oscillator is fully relativistic only in the limit as its velocity goes to zero. However, it turns out that the radiation balance at each harmonic does not depend upon the actual oscillation amplitude for small oscillations, provided that the amplitude is non-vanishing. [22] Thus the radiation equilibrium continues to hold without any change as the amplitude of oscillation goes toward zero, and the oscillator motion becomes ever closer to the relativistic limit. We expect that the radiation equilibrium obtained from approximately relativistic oscillator motion will hold even in the relativistic limit.

If one uses the action-angle variables $w$ and $J$, then the Hamiltonian in Eqs. (2) and (4) makes no reference to the mass of the oscillator or to the amplitude of oscillation, and the phase space distribution for the oscillator given in Eq. (91) becomes

$$
P_{\text {osc }}(w, J)=\text { const } \times \exp \left[-\frac{J \omega_{0}}{U_{\text {rad }}\left(\omega_{0}\right)}\right] .
$$

This phase space distribution gives equilibrium for the oscillator at all the harmonics $n \omega_{0}$ in a Lorentz-invariant radiation spectrum $U_{\text {rad }}(\omega)$. If we take the Lorentz-invariant radiation spectrum (required for full radiation equilibrium by the oscillator) as that of classical electromagnetic zero-point radiation $U_{z p r \text { zp }}(\omega)=(1 / 2) \hbar \omega$, then the oscillator phase space (17) takes the form

$$
P_{\text {osc zp }}(w, J)=\text { const } \times \exp \left[-\frac{J}{\hbar / 2}\right],
$$

where the oscillator frequency $\omega_{0}$ has cancelled out leaving a phase space which independent of frequency. This phase space distribution for the oscillator is the same as the phase space in Eq. (10) for each mode of electromagnetic radiation in the zero-point radiation field. The equilibrium phase space distribution in Eq. (18) is invariant under an adiabatic change of frequency $\omega_{0}$.

\section{SCALING IN NONRELATIVISTIC MECHANICS AND IN RELATIVISTIC ELECTRODYNAMICS}

In order to broaden our understanding of the contrasts arising in treatments of classical radiation equilibrium, we now turn to the matter of scaling. Scaling can be regarded as a 
change in the fundamental unit used to evaluate some physical quantity, or as a multiplicative change in the physical quantity itself. The contrasting scaling aspects of nonrelativistic classical mechanics and of relativistic classical electrodynamics are reflected in their determinations of thermal equilibrium.

\section{A. Scaling Aspects of Nonrelativistic Mechanics}

\section{Separate Scalings in Length, Time, and Energy}

Because nonrelativistic mechanics involves no fundamental constants, it allows separate scalings in length as $\sigma_{l}$, in time as $\sigma_{t}$, and in energy as $\sigma_{U}$. For example, any nonrelativistic classical mechanical system can be reimagined as a system where all the lengths are twice as large $\left(\sigma_{l}=2\right)$, the times are three times as long $\left(\sigma_{t}=3\right)$, and the energies are four times as great $\left(\sigma_{U}=4\right)$. This flexibility arises since the only forms of energy are kinetic energy (which can be scaled through the mass $m$ ), and potential energy $V(x, y, z)$ (which can be rescaled through the constants connecting distance to energy). For a nonlinear mechanical oscillator of mass $m$, harmonic frequency $\omega_{0}$, and nonlinear parameter $\alpha$, as in Eq. (12), the quantities $m, \omega_{0}$, and $\alpha$ are all freely adjustable, corresponding to allowing separate scalings $\sigma_{l}, \sigma_{t}, \sigma_{U}$

\section{Thermal Equilibrium Reflects the Three Separate Scalings}

Equilibrium involving the interaction of classical mechanical systems must reflect the three separate scalings allowed for nonrelativistic mechanical systems. In an equilibrium situation for nonrelativistic physics, the energy $k_{B} T$ must scale separately from the length and time parameters of the mechanical systems. If we rescale the energy (by a factor $\sigma_{U}$ ) of a mechanical system which is in equilibrium, then the equilibrium of the system should not be disturbed by the energy rescaling. Furthermore, if we replace a member of a mechanical system by a new mechanical member involving new lengths and times but with the same energy, then the mechanical equilibrium will be unchanged because the total system energy remains unchanged. If these scaling ideas are applied to the Wien-law expression in Eq. (5), they pick out only the equipartition limit involving the energy-dependent temperature $T$ but having no dependence upon the frequency-dependent $\omega$. Indeed the Boltzmann 
distribution reflects this idea of a separate energy scaling. The Boltzmann probability on phase space involves the mechanical energies of the constituent systems with no other aspect involved. Interestingly enough, the Coulomb potential (which is the only potential which can be extended to a fully relativistic classical electromagnetic theory) does not fit into the nonrelativistic Boltzmann analysis.

The Rayleigh-Jeans spectrum reflects this nonrelativistic scaling pattern where the energy $U_{\text {rad }}(\omega, T)=k_{B} T$ of each radiation mode is the same, and is entirely independent of the frequency $\omega$ of the radiation mode. It is interesting that in 1924, van Vleck[23] remarked with surprise regarding his nonrelativistic calculations that "...in a field of radiation whose specific energy $\left[\rho_{\text {rad }}(\omega, T)=\left[\omega^{2} /\left(\pi^{2} c^{3}\right)\right] U_{\text {rad }}(\omega, T)\right]$ does not vary with the frequency, we have the rather surprising result that the mean absorption is independent of the form of the force function $V(x, y, z)$ which holds the electron in the atom." Apparently, the idea of kinetic energy equipartition, completely independent of the force function $V(x, y, z)$, was a familiar and accepted idea of nonrelativistic mechanics, whereas the directly-related result associated with rates of energy absorption and loss from the radiation field treated in the dipole approximation was regarded as "surprising." Van Vleck's expressions for energy emission and absorption in the Rayleigh-Jeans spectrum can be shown to give kinetic energy equipartition. [24]

\section{No-Interaction Theorem in Relativistic Mechanics}

Many physicists are so accustomed to using nonrelativistic classical mechanics with its freedom to choose interaction potentials $V(x, y, z)$ at will, that they are surprised that relativity places very strong restrictions on systems. Indeed, most physicists have probably never heard of the "no-interaction theorem" of Currie, Jordan, and Sudarshan[4] which "says that only in the absence of direct particle interaction can Lorentz invariant systems be described in terms of the usual position coordinates and corresponding canonical momenta." [26] The standard graduate-level mechanics text then simply dismisses relativistic ideas, continuing, "The scope of the relativistic Hamiltonian framework is therefore quite limited and so for the most part we shall confine ourselves to nonrelativistic mechanics." 


\section{B. Scaling Aspects of Relativistic Electrodynamics}

\section{Single $\sigma_{l t U^{-1}-S c a l i n g}$ of Relativistic Electrodynamics}

In contrast to the three separate scalings in length, time, and energy appearing in nonrelativistic mechanics, electrodynamics allows only one single scaling connecting together length, time, and energy. This situation arises because classical electrodynamics involves several fundamental constants. Length and time are coupled through the speed of light $c$, while energy and length are coupled through the electronic charge $e$, or through Stefan's constant $a_{S}=\mathcal{U} /\left(\mathcal{V} T^{4}\right)$ related to the total thermal-part $\mathcal{U}$ of the radiation energy in a box of volume $\mathcal{V}$. Therefore (relativistic) classical electrodynamics allows only one scaling $\sigma_{l t U^{-1}}$ which preserves the values of these fundamental constants. [25] We note that Planck's constant $\hbar$ has the same dimensions as $e^{2} / c$ and so is also unchanged under $\sigma_{l t U^{-1} \text {-scaling. }}$

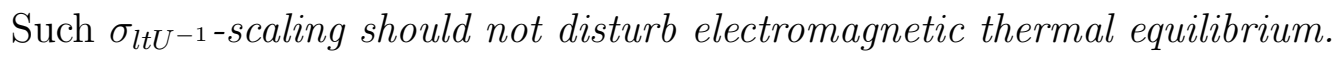

In addition to preserving the fundamental constants $c, e$, and $a_{S}$, the $\sigma_{l t U^{-1} \text {-scaling also }}$ preserves the form of Maxwell's equations. Thus invariance under $\sigma_{l t U^{-1} \text {-scaling holds }}$ for Gauss's law $\nabla \cdot \mathbf{E}=4 \pi \rho$ provided that the electric field $\mathbf{E}$ scales as charge divided by length squared. Faraday's law $\nabla \times \mathbf{E}=-(1 / c) \partial \mathbf{B} / \partial t$ satisfies invariance under the scaling provided that the magnetic field $\mathbf{B}$ also scales as charge divided by length squared. Finally, the scaling for the total energy $U$ in a volume $\mathcal{V}, U=u \mathcal{V}$, follows from the scaling for the energy density $u=[1 /(8 \pi)]\left(\mathbf{E}^{2}+\mathbf{B}^{2}\right)$.

\section{2. $\sigma_{l t U^{-1}-S c a l i n g}$ Allows a Function of $\omega / T$ for Classical Thermal Radiation}

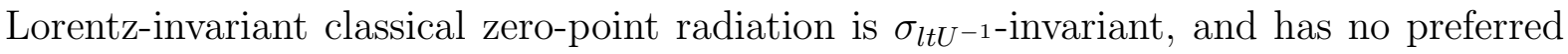
length, time, or energy.[27] Thus under a $\sigma_{l t U^{-1}}$-scale transformation, zero-point radiation is mapped onto itself. For any radiation mode of frequency $\omega$, and energy $U_{\text {rad zp }}(\omega)=$

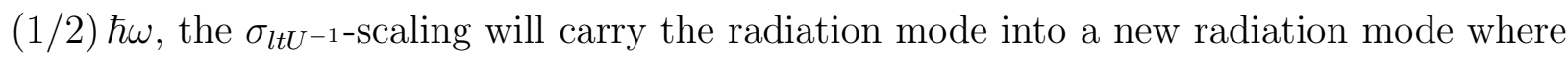
the frequency is $\omega^{\prime}=\omega / \sigma_{l t U^{-1}}$ and the energy is $U^{\prime}=U^{\prime} / \sigma_{l t U^{-1}}$. But then we have $U_{\text {rad zp }}^{\prime}\left(\omega^{\prime}\right)=U_{\text {rad zp }}(\omega) / \sigma_{l t U^{-1}}=(1 / 2) \hbar \omega / \sigma_{l t U^{-1}}=(1 / 2) \hbar \omega^{\prime}=U_{\text {rad zp }}\left(\omega^{\prime}\right)$, so that the function connecting frequency and energy is completely unchanged. Note that under this $\sigma_{l t U^{-1} \text {-scale transformation, the phase space distribution of each radiation mode remains }}$ unchanged at $P_{\text {rad zp }}\left(J_{\text {rad }}\right)=$ const $\times \exp \left[-J_{\text {rad }} /(\hbar / 2)\right] . \quad$ On the other hand, thermal 


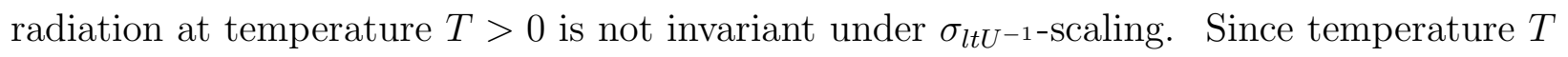
transforms as an energy, the information of Wien's law in Eq. (5) undergoes a $\sigma_{l t U^{-1} \text {-scale }}$ transformation from temperature $T$ to a new temperature $T^{\prime}=T / \sigma_{l t U^{-1}}$, while making the ratio $\omega / T$ invariant, $\omega / T=\omega^{\prime} / T^{\prime}$. Thus the $\sigma_{l t U^{-1} \text {-scaling of relativistic classical }}$ electrodynamics is consistent with Wien's law.

\section{3. $\sigma_{l t U^{-1}-S c a l i n g}$ Allows Only Zero-Point Radiation for the Relativistic Harmonic Oscillator}

Because of the existence of the fundamental constants $c$ and $e$ which require the $\sigma_{l t U^{-1-}}$ scaling of electromagnetism, a charged (relativistic) electrodynamic system in thermal radiation can have at most one freely-adjustable parameter for fixed temperature $T$. After the one parameter of the charged system has been chosen, the conditions of thermal equilibrium will determine the other physical quantities. For the (relativistic) one-dimensional harmonic oscillator with its one scaling parameter $\omega_{0}$, the oscillator energy $U_{\text {osc }}\left(\omega_{0}\right)$ is determined by the initial conditions in connection with the radiation spectrum at frequency $\omega_{0}$.

In the calculation[22] for the full electromagnetic radiation equilibrium of an oscillator of small amplitude, purely electromagnetic interactions are invoked to arrive at the same zero-point radiation spectrum which is associated with adiabatic transformation of the oscillator. However, the full Planck spectrum including zero-point radiation does not appear. Some physicists have taken this limitation as a sign that there is something wrong with the analysis, and they feel justified in their contentment with the erroneous claim that classical physics leads to the Rayleigh-Jeans spectrum. However, the reason for the limitation in the calculation is that the small-amplitude oscillator becomes a relativistic system only at zero oscillation velocity, and therefore does not show the variety of behavior of a fully

relativistic electromagnetic system. Indeed, the restriction of the (relativistic) oscillator to the zero-oscillation-velocity limit necessarily excludes velocity-dependent damping which is crucial for the low-frequency part of the Planck thermal spectrum. 
4. $\sigma_{l t U^{-1}-S c a l i n g}$ Allows a Function of $m c^{2} /\left(k_{B} T\right)$ for the Relativistic Classical Hydrogen Atom

a. Scaling for the Classical Hydrogen Atom The limiting considerations for the (approximately relativistic) harmonic oscillator do not appear for the relativistic classical hydrogen atom. The classical hydrogen atom consists of a point charge $e$ of mass $m$ in a Coulomb potential $V(r)=-e^{2} / r$ in the presence of random classical radiation. This system is fully relativistic when considered as part of classical electrodynamics. The one freely-adjustable scaling parameter is the mass $m$. Using this mass and the fundamental constants $e$ and $c$, one can form the energy $m c^{2}$, the length $e^{2} /\left(m c^{2}\right)$, and time $e^{2} /\left(m c^{3}\right)$.

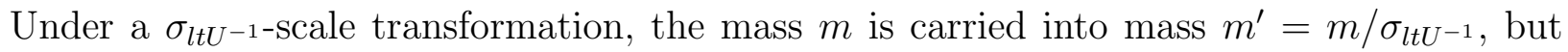

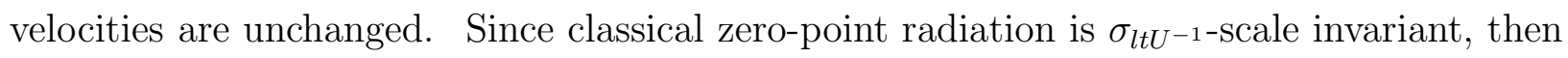
the classical hydrogen atom in zero-point radiation will have its equilibrium phase space unchanged by the transformation. Since velocities are unchanged under the transformation, this fits with the absence of velocity-dependent damping in zero-point radiation; only acceleration-dependent radiation damping is involved in equilibrium. It is familiar that for circular orbits, the speed of a particle in a circular orbit is $v=e^{2} / J$ where $J$ is the angular

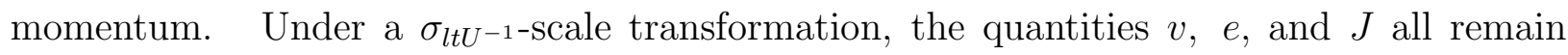
unchanged.

b. Scaling for Hydrogen in Zero-Point Radiation In zero-point radiation, the scale of the random radiation is given by $\hbar$ which has the same dimensions as $e^{2} / c$. Thus in the limit which removes all factors of $c$, we find that the classical hydrogen atom in zero-point radiation has a typical length $\left[e^{2} /\left(m c^{2}\right)\right]\left[\hbar c / e^{2}\right]^{2}=\hbar^{2} /\left(m e^{2}\right)$, a typical time $\left[e^{2} /\left(m c^{3}\right)\right]\left[\hbar c / e^{2}\right]^{3}=\hbar^{3} /\left(m e^{4}\right)$, and a typical energy $m c^{2}\left[e^{2} /(\hbar c)\right]^{2}=m e^{4} / \hbar^{2}$. These quantities, which involve no factors of $c$, are familiar from the Bohr model of hydrogen. They also appear in work involving both numerical simulations and the Fokker-Planck equation for the classical hydrogen atom in classical zero-point radiation. [28]

c. Scaling for Hydrogen in Thermal Radiation with $T>0$ If the classical hydrogen atom is in equilibrium with thermal radiation at temperature $T>0$, then there will be

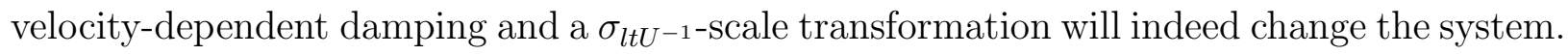
The relativistic particle in a Coulomb potential allows a phase space distribution dependent upon both the mass $m$, and also the fundamental constants $e$ and $c$. Thus the phase space 
for the Coulomb situation can involve the characteristic energy ratio $m c^{2} /\left(k_{B} T\right)$ which is freely adjustable in both $m$ and in $T$. This freely-adjustable ratio allows the possibility of a transition between a high-frequency region dominated by acceleration-dependent radiation damping and a low-frequency region dominated by velocity-dependent damping.

For the Coulomb potential, when the orbital radius is small, the velocity is high and the frequency is high, corresponding to the zero-point radiation part of the spectrum where acceleration-dependent radiation damping dominates and velocity-dependent damping is minor. On the other hand, when the orbital radius is large, the velocity and frequency are low, and acceleration-based radiation damping is also low. In this low-frequency region, velocity-dependent damping due to thermal radiation for $T>0$ might well dominate. This situation can be illustrated[29] by considering relativistic circular orbits characterized by angular momentum $J$ where the velocity is $v=e^{2} / J$, the energy is $U(J)=m c^{2} \sqrt{1-\left[e^{2} /(J c)\right]^{2}}$, the radius is $r=\left[e^{2} /\left(m c^{2}\right)\right]\left(J c / e^{2}\right)^{2} \sqrt{1-\left[e^{2} /\left(J_{c}\right)\right]^{2}}$, and the frequency is $\omega=\partial U / \partial J=\left(m c^{3} / e^{2}\right)\left[e^{2} /(J c)\right]^{3} / \sqrt{1-\left[e^{2} /(J c)\right]^{2}}$. The ratio of orbital frequency $\omega$ to temperature $T$ can be written as

$$
\frac{\hbar \omega}{k_{B} T}=\frac{m c^{2}}{k_{B} T}\left(\frac{\hbar c}{e^{2}}\right)\left(1-\left(\frac{e^{2}}{J c}\right)^{2}\right)^{-1 / 2}\left(\frac{e^{2}}{J c}\right)^{3} .
$$

We see from Eq. (19), that for changing temperature $T$ and for fixed mass $m$ and fixed angular momentum $J$ (which corresponds to fixed frequency $\omega$ ), the system changes from the high-frequency region of the thermal spectrum $\hbar \omega / k_{B} T>>1$, to the low-frequency region $\hbar \omega / k_{B} T<<1$ exactly in unison with the ratio $m c^{2} /\left(k_{B} T\right)$. The transition between the two regions of behavior is mediated by the ratio $m c^{2} /\left(k_{B} T\right)$. An analogous ratio does not exist for the (approximately relativistic) harmonic oscillator.

\section{MISCELLANEOUS ASPECTS OF THERMAL EQUILIBRIUM}

\section{A. Full Thermal Equilibrium Requires Both Velocity-Dependent Damping and} Acceleration-Dependent Damping

Indeed it is interesting to see that within classical physics, thermal radiation equilibrium for non-zero temperature $T>0$ requires both acceleration-dependent radiation damping and velocity-dependent damping. The Einstein-Hopf analysis[14][15] of 1910 considered a 
particle of large mass $M$ containing an electric dipole oscillator which experienced velocitydependent damping when moving through random radiation, but there were no non-radiation forces and so the particle motion experienced no acceleration-dependent damping. Assuming that the average kinetic energy of the one-dimensional motion of the large mass $M$ was $(1 / 2) k_{B} T$, the Einstein-Hopf analysis led to the low-frequency, velocity-dependent, Rayleigh-Jeans part of the Planck law for thermal radiation equilibrium. Indeed, the velocity-dependent damping without acceleration-dependent radiation damping is analogous to the situation which arises in nonrelativistic mechanics where thermal equilibrium depends entirely upon velocity-dependent damping, and no acceleration-dependent damping can appear. It was only later when the Einstein-Hopf analysis was extended[30] to also include acceleration-dependent radiation damping of the particle motion (arising from non-radiation forces on the particle) that the classical Einstein-Hopf analysis led to the full Planck spectrum including zero-point radiation. Furthermore, the inclusion of classical zeropoint radiation in the Planck spectrum suggests the possibility of superfluid-like behavior for an Einstein-Hopf particle at low temperatures. [31]

\section{B. Information Contained within the Rayleigh-Jeans and Zero-Point Radiation} Spectra

\section{Zero-Point Spectrum Has Least Information}

Zero-point radiation involves the spectrum of random radiation with least possible information. The spectrum is Lorentz invariant and has no preferred inertial frame, and hence no velocity-dependent damping. The spectrum is also scale invariant, and has no preferred length or time or energy. Even in curved spacetime, zero-point radiation has correlation functions which involve only the geodesic separations between the spacetime points where the correlation is evaluated.

\section{Information in the Rayleigh-Jeans Spectrum}

The Rayleigh-Jeans spectrum contains more information than the classical zero-point radiation spectrum. Both spectra are characterized by a single parameter: $\hbar$ for zero-point 
radiation and $T$ for the Rayleigh-Jeans spectrum. For the Rayleigh-Jeans spectrum, the temperature $T$ is freely-adjustable, and the energy per normal mode is $U_{\text {rad }} \mathrm{RJ}(\omega, T)=k_{B} T$ at any frequency $\omega$. However, the Rayleigh-Jeans spectrum determines exactly one preferred inertial frame in which it is isotropic. Furthermore, at fixed temperature $T$, one can determine whether one frequency is larger than another frequency by comparing their phase space distributions. Thus the phase space distribution for electromagnetic radiation in the Rayleigh-Jeans spectrum is $P_{\text {rad RJ }}(J, \omega, T)=$ const $\times \exp \left[-J \omega /\left(k_{B} T\right)\right]$ so that a larger value of frequency $\omega$ corresponds to a phase space distribution more concentrated near $J=0$. On the other hand, the classical zero-point radiation spectrum $U_{\text {rad zp }}(\omega)=(1 / 2) \hbar \omega$ has a phase space distribution which is the same for radiation modes of any frequency $\omega$, $P_{\text {rad zp }}(J, \omega)=$ const $\times \exp [-J /(\hbar / 2)]$. Zero-point radiation has no preferred inertial frame and is isotropic in every inertial frame. If a harmonic oscillator of small amplitude were to scatter zero-point radiation so as to enforce any radiation spectrum other than the zeropoint radiation spectrum, the oscillator would impose a preferred inertial frame upon the scattered radiation.

3. Thermal Radiation Spectrum as Giving Least Information in the Presence of Zero-Point Radiation

Thermal radiation at $T>0$ involves a finite amount of energy spread over the modes of the zero-point radiation spectrum and so introduces a preferred inertial frame. The spectrum of zero-point radiation acts as a noise spectrum into which the finite amount of thermal energy is introduced, and the spectrum of thermal radiation represents the minimal information consistent with the finite total available thermal energy and the divergent Lorentz-invariant spectrum of zero-point radiation. The entropy of thermal radiation at

frequency $\omega$ is determined by comparing the amount of thermal radiation to the amount of zero-point radiation at that frequency. [3]

\section{Inconsistent Mixtures of Nonrelativistic and Relativistic Physics}

The derivations of the Rayleigh-Jeans law for thermal radiation given in the textbook literature arise from attempts to combine nonrelativistic mechanics with relativistic elec- 
trodynamics. Such inconsistent mixtures of nonrelativistic and relativistic theories satisfy neither Galilean invariance nor Lorentz invariance. Indeed, the results of the analysis depend upon the inertial frame in which the analysis is done. For particles undergoing point collisions, this situation has already been exhibited explicitly in the literature.[32] Thus if one considers the point collision between two particles, using relativistic physics for one and nonrelativistic physics for the other, one can solve for the motion of the particles after the collision by using the energy and momentum conservation laws in any inertial frame; however, the results satisfy neither the nonrelativistic conservation law for the uniform motion of the center of mass nor the relativistic conservation law for the uniform motion of the center of energy, and the results indeed depend upon the inertial frame chosen for the analysis.

\section{CLOSING SUMMARY}

\section{A. Two Natural Spectra Associated with Harmonic Oscillators}

Associated with harmonic oscillators, there are two spectra of random radiation which arise in a natural manner. One of these is the Rayleigh-Jeans spectrum which is associated with the equipartition ideas of nonrelativistic classical mechanics and is based upon velocitydependent damping. The other is the classical zero-point radiation spectrum which arises from an adiabatic transformation of oscillator frequency and is associated with relativistic classical electrodynamics which includes acceleration-dependent damping.

\section{B. Oscillators in Translational Motion and Two Forms of Damping}

If one considers not a stationary oscillator but rather an oscillator which is free to move in over-all translation, then one can obtain either the Rayleigh-Jeans spectrum (obtained for a situation with velocity-dependent damping on the oscillator but no acceleration-dependent

damping by Einstein and Hopf[14]) or the full Planck spectrum with zero-point radiation (if one allows both types of damping on the oscillator[30]). 


\section{Thermal Radiation Equilibrium from Scattering}

A charged harmonic oscillator at rest with its electromagnetic interactions limited to the dipole approximation is in equilibrium with any spectrum of radiation; the energy of the oscillator merely matches the energy of the radiation modes at the natural frequency of the oscillator. If we wish to go beyond this ambiguous radiation situation and to determine a preferred equilibrium radiation spectrum under scattering, then there are two natural scattering extensions. If we continue the limitation on the electromagnetic interactions to the dipole approximation but go to a more complex nonrelativistic mechanical motion, then we arrive at the Rayleigh-Jeans spectrum. On the other hand, if we treat the charged harmonic oscillator as a fully electromagnetic system but with only approximately-relativistic harmonic oscillator mechanical behavior, then we arrive at classical zero-point radiation as the preferred radiation spectrum. Scattering by a fully relativistic electromagnetic system, such as the classical hydrogen atom, has the possibility of giving the full Planck radiation spectrum with zero-point radiation as the equilibrium classical radiation spectrum.

\section{Relativity and Thermal Radiation}

If one reads James Jeans' Report on Radiation and the Quantum-Theory published in 1914 or if one reads the modern physics textbooks published in the last few years, one would find no inkling that special relativity might have some relevance to the blackbody radiation problem. However, the equilibrium radiation spectrum is determined by assumptions on both the mechanical motion (whether nonrelativistic or relativistic) and the relativistic electromagnetic interactions. In any case, the radiation equilibrium of the fully electromagnetic charged harmonic oscillator of small amplitude does not lead to the Rayleigh-Jeans spectrum. It is an outright error to claim that classical physics leads inevitably to the Rayleigh-Jeans spectrum.

[1] See for example, R. Eisberg and R. Resnick, Quantum Physics of Atoms, Molecules, Solids, Nuclei, and Particles 2nd ed. (Wiley, New York 1985); K. S. Krane, Modern Physics, 2nd ed. (Wiley, New York 1996); R. Taylor, C. D. Zafiratos, and M. A. Dubson, Modern Physics 
for Scientists and Engineers, 2nd ed. (Pearson, New York, 2003); S. T. Thornton and A. Rex, Modern Physics for Scientists and Engineers, 4th ed. (Brooks/Cole, Cengage Learning, Boston, MA, 2013).

[2] J. H. Jeans, Report on Radiation and the Quantum Theory (www.ForgottenBooks.org, 2013). This is a reproduction from the report originally published in 1914.

[3] T. H. Boyer, "Blackbody radiation in classical physics: A historical perspective," Am. J. Phys. 86, 495-509 (2018).

[4] D. G. Currie, T. F. Jordan, and E. C. G. Sudarshan, "Relativistic Invariance and Hamiltonian theories of interacting particles," Rev. Mod. Phys. 35, 350-375 (1963).

[5] T. H. Boyer, "Thermodynamics of the harmonic oscillator: Wien's displacement law and the Planck spectrum," Am. J. Phys. 71, 866-870 (2003).

[6] T. H. Boyer, "Thermodynamics of the harmonic oscillator: derivation of the Planck blackbody spectrum from pure thermodynamics," Eur. J. Phys. 40, 025101(16pp) (2019).

[7] See for example, M. Planck, The Theory of Heat Radiation (Dover, New York 1959).

[8] T. W. Marshall, "Random electrodynamics," Proc. R. Soc. A276, 475-491 (1963).

[9] T. H. Boyer, "Random electrodynamics: The theory of classical electrodynamics with classical electromagnetic zero-point radiation," Phys. Rev. D 11, 790-808 (1975).

[10] B. H. Lavenda, Statistical Physics: A Probabilistic Approach (Wiley, New York 1991), pp. 73-74.

[11] T. H. Boyer, "Understanding zero-point energy in the context of classical electromagnetism," Eur. J. Phys. 37, 055206(14) (2016).

[12] A review of the work on classical electromagnetic zero-point radiation up to 1996 is provided by L. de la Pena and A. M. Cetto, The Quantum Dice - An Introduction to Stochastic Electrodynamics (Kluwer Academic, Dordrecht 1996). For a more recent short review, see T. H. Boyer, "Stochastic Electrodynamics: The Closest Classical Approximation to Quantum Theory," Atoms 7(1), 29-39 (2019).

[13] T. H. Boyer, "The contrasting roles of Planck's constant in classical and quantum theories," Am. J. Phys. 86, 280-283 (2018).

[14] A. Einstein and L. Hopf, "Statistische Untersuchung der Bewegung eines Resonators in einem Strahlungsfeld," Annalen der Physik (Leipzig) 33, 1105-1115 (1910).

[15] For modern notation, see P. W. Milonni, The Quantum Vacuum: An Introduction to Quantum 
Electrodynamics (Academic Press, Boston1994), pp. 11-14.

[16] J. H. van Vleck, "The absorption of radiation by multiply periodic orbits, and its relation to the correspondence principle and the Rayleigh-Jeans law: Part II. Calculation of absorption by multiply periodic orbits," Phys. Rev. 24, 347-365 (1924); "A correspondence principle for absorption," Jour. Opt. Soc. Amer. 9, 27-30 (1924).

[17] T. H. Boyer, "Equilibrium of random classical electromagnetic radiation in the presence of a nonrelativistic nonlinear electric dipole oscillator," Phys. Rev. D 13, 2832-2845 (1976).

[18] R. Blanco, L. Pesquera, and E. Santos, "Equilibrium between radiation and matter for classical relativistic multiperiodic systems. Derivation of Maxwell-Boltzmann distribution from Rayleigh-Jeans spectrum," Phys. Rev. D27, 1254-1287 (1983); "Equilibrium between radiation and matter for classical relativistic multiperiodic systems. II. Study of radiative equilibrium with Rayleigh-Jeans radiation," Phys. Rev. D 29, 2240-2254 (1984).

[19] M. Born, The Mechanics of the Atom (Ungar, New York 1970), pp. 66-71.

[20] J. D. Jackson, Classical Electrodynamics 3rd ed. (John Wiley \& Sons, New York, 1999), p. 704, problem 14.22.

[21] W. C-W. Huang and H. Batelaan, "Discrete Excitation Spectrum of a Classical Harmonic Oscillator in Zero-Point Radiation," Found. Phys. 45, 333-353 (2015).

[22] T. H. Boyer, "Equilibrium for classical zero-point radiation: detailed balance under scattering by a classical charged harmonic oscillator," J. Phys. Commun. 2, 105014(17) (2018).

[23] See ref. 16, p. 359. Van Vleck's comment involves radiation absorption but is related to nonrelativistic kinetic energy and to the nonrelativistic Larmor formula for radiation emission. Thus for a general oscillation such as suggested by our Eq. (14), the average kinetic energy in the $n^{\text {th }}$ harmonic involves $\left(n \omega_{1}\right)^{2} D_{n}^{2}$ whereas the average radiation emission by the $n^{\text {th }}$ harmonic is $\left(n \omega_{1}\right)^{2}$ times as large, involving $\left(n \omega_{1}\right)^{4} D_{n}^{2}=\left(n \omega_{1}\right)^{2}\left[\left(n \omega_{1}\right)^{2} D_{n}^{2}\right]$.

[24] T. H. Boyer, "Statistical equilibrium of nonrelativistic multiply periodic classical systems and random classical electromagnetic radiation," Phys. Rev. A 18, 1228-1237 (1978).

[25] T. H. Boyer, "Scaling symmetries of scatterers of classical zero-point radiation," J. Phys. A: Math. Theor. 40, 9635-9642 (2007); "Scaling symmetry and thermodynamic equilibrium for classical electromagnetic radiation," Found. Phys. 19, 1371-1383 (1989).

[26] H. Goldstein, C. Poole, and J. Safko, Classical Mechanics 3rd ed. (Addison-Wesley, New York, 2002), p. 353. 
[27] T. H. Boyer, "Conformal Symmetry of Classical Electromagnetic Zero-Point Radiation," Found. Phys. 19, 349-365 (1989).

[28] For work on the classical hydrogen atom in classical zero-point radiation, see D. C. Cole and Y. Zou, "Quantum Mechanical Ground State of Hydrogen Obtained from Classical Electrodynamics," Phys. Lett. A 317, 14-20 (2003), and T. H. Boyer, "Relativity and Radiation Balance for the Classical Hydrogen Atom in Classical Electromagnetic Zero-Point Radiation,” Eur. J. Phys. 42, 025205 (24pp) (2021).

[29] T. H. Boyer, "Unfamiliar trajectories for a relativistic particle in a Kepler or Coulomb potential," Am. J. Phys. 75, 992-997 (2004).

[30] T. H. Boyer, "Derivation of the Blackbody Radiation Spectrum without Quantum Assumptions," Phys. Rev. 182, 1374-1383 (1969).

[31] T. H. Boyer, "Particle Brownian motion due to random classical radiation: Superfluid-like behavior in classical zero-point radiation," Eur. J. Phys. 41, 055103 (17pp) (2020).

[32] T. H. Boyer, "Illustrating some implications of the conservation laws in relativistic mechanics," Am. J. Phys. 77, 562-569 (2009). 